\title{
Mise en scène du discours d'autorité des experts dans la publicité. De l'éloquence du scientifique à la maladresse du praticien
}

Staging advertising experts' discourse of authority. From the scientist's eloquence to the practioner's missteps

La puesta en escena del discurso de autoridad de los expertos en la publicidad. Desde la elocuencia del científico a la torpeza del practicante

\section{Gaëlle Planchenault}

\section{OpenEdition \\ Journals}

Édition électronique

URL : https://journals.openedition.org/mots/21725

DOI : $10.4000 /$ mots. 21725

ISSN : 1960-6001

Éditeur

ENS Éditions

Édition imprimée

Date de publication : 15 septembre 2014

Pagination : 105-126

ISBN : 978-2-84788-543-9

ISSN : 0243-6450

\section{Référence électronique}

Gaëlle Planchenault, « Mise en scène du discours d'autorité des experts dans la publicité. De

l'éloquence du scientifique à la maladresse du praticien », Mots. Les langages du politique [En ligne],

105 | 2014, mis en ligne le 15 septembre 2016, consulté le 23 avril 2022. URL : http://

journals.openedition.org/mots/21725; DOI : https://doi.org/10.4000/mots.21725 


\title{
Mise en scène du discours d'autorité des experts dans la publicité. De l'éloquence du scientifique à la maladresse du praticien
}

\author{
On distinguait traditionnellement deux profils : \\ l'intellectuel et le politique ou, le savant et le politique. \\ Aujourd'hui, pour des raisons structurelles, et non \\ conjoncturelles, apparaît ce troisième profil. \\ L'expert emprunte aux deux modèles. Pour l'analyse, \\ il utilise les connaissances, les réflexes de l'intellectuel. \\ Pour l'action, il est lié à l'acteur politique. \\ (Wolton, 1995)
}

S'il existe une similitude notable entre les imaginaires des discours politiques et publicitaires, il s'agit bien de la relation complexe que ces discours entretiennent avec les notions de vérité et d'autorité : qui parle, au nom de qui et en référence à quelle réalité ? Ainsi, la journaliste Anne-Laure Gannac, présentant un numéro du magazine Psychologie ${ }^{1}$ consacré au parler-vrai, soulignait-elle : «Le but du politique, c'est d'inspirer confiance [...] Et il ne peut y avoir de relation de confiance sans une parole vraie, c'est-à-dire sans une certaine garantie que ce que l'autre nous dit est au plus près de sa vérité, de ce qu'il ressent comme une vérité. » Alors que "des logiques analogues rapprochent les discours publicitaire et politique : ce sont deux types de "discours d'influence" ou "persuasifs", dont l'objectif est d'agir sur les destinataires [...]» (Barbet, Desmarchelier, 2012, p. 7), s'est développé un sentiment de méfiance vis-à-vis de rhétoriques dont le but affiché est de séduire et de convaincre. Afin de retrouver la confiance perdue des consommateurs et des électeurs, on a vu s'affirmer la nécessité d'avoir recours à des experts mais aussi, alors que la nuance entre "se tromper» et «tromper» semblait toujours s'estomper davantage, celle de vérifier (ou invalider) les preuves sur lesquelles leurs paroles s’appuient. Un

1. http://www.franceinfo.fr/decryptage/modes-de-vie/parler-vrai-en-politique-567867-2012-03-26 (consulté le 6 mai 2014, de même que tous les sites indiqués dans les notes, sauf mention contraire). 
autre point commun des discours publicitaires et politiques aura à voir avec la mise en scène de la parole d'autorité qui y prend place. Rarement improvisés mais intervenant dans des cadres définis, et plus particulièrement sur des scènes politiques ou médiatiques, ces discours sont à interpréter en fonction de ces cadres et selon des règles de genre (qu'il s'agisse du discours, du slogan ou de la réclame). Dans cet article, je soutiendrai que la parole d'autorité est elle-même un genre qui, afin d'être reconnaissable comme telle, obéit à des règles discursives et linguistiques (phonétiques, prosodiques, lexicales et syntaxiques), tout en incluant dans une certaine mesure des variations qui sont fonction des types de locuteurs et de locutrices.

Pour revenir au cadre qui nous intéresse dans la présente étude, constatons que pas une semaine ne passe sans que le mot expert ne s'affiche dans les publicités (des produits de beauté L'Oréal Men Expert aux dentifrices Oral-B Pro-Expert, Colgate Total Expert Nettoyage en passant par divers aliments pour chiens ou produits ménagers). Selon Jean-Yves Trépos (1996, p.3), «[l]e mot « expert» connaît une vogue indéniable [...] la presse mentionne volontiers le recours, dans une situation exceptionnelle ou délicate, à tel spécialiste, qu'elle qualifie d'expert [...]». Et si pour le Petit Robert, l'expert est la «personne choisie pour ses connaissances techniques et chargée de faire, en vue de la solution d'un procès, des examens, constatations ou appréciations de fait », force est de constater que les médias montrent un large éventail de ces voix d'expertise. Mais qui sont les experts qui se cachent derrière marques et slogans? Comme le rappelle J.-Y. Trépos (ibid.), « plus généralement, on qualifie d'expert des personnes auxquelles on accorde une virtuosité particulière, quelquefois même en dehors de tout acte de travail». Ainsi, dans les publicités, les différentes voix d'experts comptent les traditionnels scientifiques, techniciens ou professionnels d'ordre divers, mais incluent également les voix de non-professionnels (mères de famille, propriétaires d'animaux, etc.). Est alors expert celui ou celle qui connait bien la situation dans laquelle l'emploi du produit est recommandé ou utilise ce produit régulièrement. Tenant compte de l'aspect polysémiotique caractéristique des publicités télévisées (dans lesquelles le sens est fourni aussi bien par le texte que par l'image, c'est-à-dire par les choix de mise en scène et le jeu des acteurs), nous proposons une analyse linguistique et multimodale de trois publicités qui nous permettra de mettre en lumière les imaginaires langagiers liés aux paroles d'experts dans la publicité. Cette analyse situera les différentes variétés de ces imaginaires sur plusieurs axes : (relâché $\rightarrow$ énergique) ; (maladroit $\rightarrow$ éloquent) ; (authentique $\rightarrow$ mis en scène). 


\section{Statut de l'expert : rapport à la vérité et qualité de la parole d'autorité}

Alors que leurs statuts ne s'excluent pas nécessairement, on a traditionnellement opposé l'expert au scientifique. Ainsi Petit (2000, p. 7) note-t-il une divergence de fonctions :

Un expert n'est pas a priori un scientifique (et réciproquement) du fait de la finalité de leurs fonctions [...] ; alors que l'expert agit sur requête (le site énonciatif de celleci étant situé dans un autre domaine), le scientifique mène une recherche à l'intérieur de son domaine, même s'il entreprend une activité de vulgarisation.

Le passage du scientifique à l'expert se fait par l'investissement dans une tâche de constatation, certification ou recommandation, mandatée par des instances externes au milieu scientifique ou académique. Le discours de l'expert est parole d'autorité dans la mesure où il entretient des liens forts, d'une part, avec le savoir et, d'autre part, avec la vérité (à laquelle l'expert est tenu). Ainsi, selon Gérard Petit toujours, pour le scientifique et l'expert :

Le rapport au savoir et à la vérité diffère également : le savoir du scientifique peut être en suspens sur un point sans que cela n'entraîne pour lui une déqualification; un expert, en revanche, doit résoudre un suspens; il est censé disposer d'un savoir qui conduit à la production de la vérité (de fait il est supposé dire le vrai). (Petit, 2000, p.7)

Dans la publicité, le recours aux scientifiques et aux experts n'est ni récent ni rare (les publicités que nous analysons dans cet article proposent des mises en scène des deux types d'intervenants). Toutefois, ceux ou celles qui apparaissent dans les spots télévisés sont rarement de vrais scientifiques mais plutôt des acteurs et des actrices à qui il est demandé de jouer des archétypes du savoir et de l'expertise. Pire encore, il n'est pas toujours aisé de distinguer les vrais scientifiques/experts des comédiens tant les publicitaires se plaisent à maintenir les téléspectateurs dans le flou. Afin d'éviter les dérives, des codes de déontologie ont été créés dès la première moitié $d u 20^{e}$ siècle. Ainsi depuis 1937, le CODE ICC de la Chambre de commerce internationale définit normes et règles de bonne conduite. Voici, par exemple, un extrait du CODE ICC consolidé sur les pratiques de publicité et de communication commerciale $\left(2011\right.$, p. 8) ${ }^{2}$ qui réglemente le devoir de véracité de l’information commerciale ainsi que l'usage qui y est fait du discours scientifique :

2. http://www.icc-france.fr/chambre-de-commerce-internationale-page-3-60-19o-Code_consolide_sur_la_publicite.html. 


\title{
ARTICLE 5 - Véracité
}

La communication commerciale doit être véridique et ne peut être trompeuse 3 .

\begin{abstract}
ARTICLE 6 - Utilisation de données et d'une terminologie techniques/scientifiques La communication commerciale ne doit pas :

- abuser de données techniques, par exemple, des résultats de recherches ou des extraits de publications techniques et scientifiques;

- présenter de statistiques de manière à exagérer l'applicabilité d'une allégation relative à un produit;

- utiliser une terminologie ou un vocabulaire scientifiques de manière à suggérer à tort qu'une allégation relative à un produit a un fondement scientifique.

(CODE ICC consolidé sur les pratiques de publicité et de communication commerciale, 2011, p. 8)
\end{abstract}

En ce qui concerne les publicités formulant des allégations santé, l'Autorité de régulation professionnelle de la publicité (ARPP) présente ces avertissements 4 :

2 3.1 Une recommandation, émanant d'un ou des membre(s) d'une profession médicale, paramédicale ou scientifique, peut s'appliquer au composant d'un produit, sous réserve qu'elle repose sur des preuves scientifiques objectives et vérifiables et qu'elle ne reflète pas seulement l'opinion personnelle du ou des professionnels représentés.

2 3.2 Ces messages ne doivent pas conférer une présentation médicale au produit.

2 3.3 La recommandation directe d'un produit n'est pas acceptée. (ARPP, 2002)

Au vu de ces avertissements, on voit émerger un paradoxe qui veut que celui qui est mandaté en tant qu'expert se cantonne à la partie constative de sa tâche et se garde d'émettre des conseils. Il ne doit donc pas s'engager personnellement quant à la parole qu'il délivre. Remarquons pourtant que hors du discours publicitaire, les experts sont impliqués par leurs discours d'expertise et que, juridiquement, ils peuvent être tenus responsables par des juges.

Parmi d'autres traits saillants du discours d'autorité, nos analyses s'intéresseront au niveau d'énergie phonatoire et à l'articulation des participants. Dans son livre sur la voix et s'appuyant sur les travaux de psychologues américains sur les émotions, Joana Revis (2013, p. 162-163) définit trois types de phonation dont chacun est fonction d'un niveau d'activation psycho-physiologique (arousal) : la voix minimale, la voix neutre et la voix soutenue. Selon ces types, on argumentera que la phonation utilisée par maints professionnels du petit écran (journalistes et présentateurs), et particulièrement de la publicité5, pré-

3. Rappelons par ailleurs que, selon l'article L121-1 du Code de la consommation, la loi interdit «toute publicité comportant, sous quelque forme que ce soit, des allégations, indications ou présentations fausses ou de nature à induire en erreur » (http://www.legifrance.gouv.fr/).

4. http://www.arpp-pub.org/IMG/pdf/Allegations_sante.pdf

5. L'auteure de cet article a conscience du postulat sur lequel s'appuie la présente étude, qui repose davantage sur un constat que sur une évaluation empirique (laquelle, en raison de la largeur du corpus à analyser, aurait dépassé les limites du cadre de cette recherche). 
sente des variations importantes entre le niveau neutre (voix conversationnelle) et celui de la voix soutenue : «mobilis[ant] davantage l'effort », "c'est la voix de l'enseignant, du comédien de théâtre, du conférencier, du chef d'entreprise en réunion, etc. C'est la voix de l'autorité ou de la prise de parole en public » (Revis, 2013, p. 163 [nous soulignons]). À ce niveau, il s'agit pour les locuteurs de faire passer un message et d'éviter tout risque d'incompréhension, mais aussi, si possible, de convaincre et de séduire par le déploiement d'une virtuosité phonatoire. Une part des analyses qui vont suivre s'intéressera également à l'articulation, concept qui sera pris non pas dans le sens phonétique du terme, mais plutôt comme trait de l'imaginaire linguistique du français (Planchenault, 2012). En effet, notre recherche passée sur les vernaculaires non-standards dans les médias et les pratiques discursives du professeur d'université a mis au jour une opposition binaire nette entre l'imaginaire d'une bonne articulation (indexant éloquence, contrôle de soi, maitrise intellectuelle mais aussi artifice et distance) et celle d'une articulation relâchée (indexant tantôt manque de savoir-vivre ou déficience intellectuelle, tantôt proximité et authenticité).

\section{Analyse de la mise en scène publicitaire des paroles d'experts}

Ce travail d'analyse se fait principalement sur deux publicités : la publicité Oral-B Pro-Expert sur M6 (2012) et la publicité Calgon sur France 5 (2013). Si la première publicité met en scène une véritable experte, la deuxième présente des acteurs qui jouent les rôles d'experts (seul le présentateur joue son propre rôle). Ainsi, notons que ces publicités mettent en scène trois catégories d'experts :

- des expertsscientifiques: nommés ainsi dans la publicité (« expertOral-B») ${ }^{6}$ ou par le biais de termes co-référentiels : «chercheur », « scientifique(s) », « hydrologue», etc. ;

- des journalistes : également considérés comme des experts, ils sont présentés comme tels par les chaines qui les emploient. Ainsi, M6 fournit-elle les informations suivantes sur son site : «M6 Publicité et Oral B ont conçu un dispositif reposant sur la caution scientifique de l'animateur Mac Lesggy. Particulièrement légitime, via son expertise au sein de son émission $\mathrm{E}=\mathrm{M} 6[\ldots]$...] 7 [nous soulignons]. Voici maintenant comment Stéphane Thebaut est présenté sur le site de l'émission qu'il anime sur la $5^{\mathrm{e}}$ chaine : "Idées pratiques, conseils avisés, dernières tendances [...] Les amateurs de déco, mais également les plus indécis ou les moins inspirés peuvent compter sur Stéphane Thebaut pour les

\footnotetext{
6. Titre choisi pour identifier la scientifique, plutôt que le réel intitulé de son poste (chefde projet Recherche et Santé Procter et Gamble). Ce choix a-t-il été fait par souci de cohérence avec le nom du produit? Par ailleurs, pourquoi a-t-on préféré la forme masculine plutôt que le féminin « experte» ?

7. http://m6pub.fr/operation-speciale/oral-b-et-mac-lesggy/.
} 
aider à repenser entièrement l'univers de leur habitat ${ }^{8}$ [nous soulignons]. Nous ajouterons ainsi que l'expertise de ces présentateurs tient aussi à leur compétence à s'entourer d'experts dans différents domaines;

- autres : professionnels mais aussi non-professionnels, ils ne sont pas experts au sens strict du terme (ni présentés comme tels par les publicités), mais réparateurs (ils ont alors «l'expertise de ceux qu'il y a peu encore on appelait des “pros” »-Trépos, 2000) ou mères de famille par exemple. Leur expertise vient de leur connaissance rapprochée du produit (ils l'utilisent régulièrement / depuis longtemps) ou des conditions particulières dans lesquelles une utilisation du produit est recommandée. On remarque ainsi que si les catégories précédentes sont liées à la connaissance et au savoir, cette catégorie relève de l'expérience et renvoie donc au sens premier du mot expert (du latin expertis qui désigne l'épreuve, et par extension celui ou celle qui a éprouvé).

Dans les analyses qui suivent, nous verrons que chaque catégorie s'attache à une mise en scène particulière de la parole d'autorité et suit des règles discursives précises. Chaque transcription' 9 des publicités sera suivie d'une analyse multimodale qui inclura aussi bien une analyse linguistique des dialogues qu'une analyse de la gestuelle, des éléments de direction ou de la mise en scène (costumes, accessoires, types de plans ${ }^{10}$, montage, etc.).

\section{La publicité Oral-B Pro-Expertsur M6}

Sur le plateau de l'émission $E=M 6^{11}$, le journaliste Mac Lessgy (ML dans les transcriptions) est d'abord au premier plan puis au côté de Charlotte Dumézy (CD dans les transcriptions) en blouse blanche et lunettes qui se tient debout derrière une table haute de type paillasse de laboratoire et devant un mur écran sur lequel apparait une liste d'éléments - on peut lire : attaques acides, gencives, plaque dentaire, dents sensibles, émail, tartre, blancheur, haleine fraiche.

Le style de Mac Lessgy se définit par une dépense d'énergie élevée. Sur l'échelle de niveaux phonatoires définie par Revis (2013, p.163), nous estimons que la voix utilisée par le présentateur s'inscrit dans un niveau soutenu. Ce style discursif est à lier au genre médiatique (c'est-à-dire au style adopté par certains présentateurs de télévision ${ }^{12}$, en particulier ceux de la chaine M6) mais aussi au personnage d'enthousiaste que joue Mac Lessgy dans les émissions $E=M 6$. On trouve cette énergie sur le plan visuel aussi bien que sonore où une

8. http://www.france5.fr/emissions/la-maison-france-5/biographies.

9. Les transcriptions réalisées pour cet article suivent la Convention ICOR (voir en annexe).

10. Pour plus de détails sur le vocabulaire technique, voir Vanoye et Goliot-Lété (1992, p. 28-29).

11. http://m6pub.fr/operation-speciale/oral-b-et-mac-lesggy/.

12. Alice Coffin (2013) dénonce ainsi des tics discursifs ("voix mécaniques», "intonations artificielles», «stéréotypes d'expression », «manie des phrases descendantes») qui finissent paragacer. 
Tableau 1. La publicité Oral-B Pro-Expert sur M6

\begin{tabular}{|c|c|}
\hline AUDIO & VISUEL \\
\hline 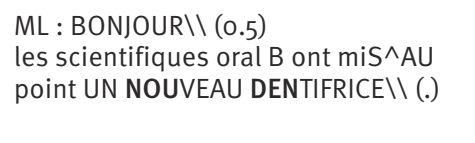 & $\begin{array}{l}\text { - zoom sur ML en pied, bras ouverts } \\
\text { - ramenant les mains vers le corps, poings } \\
\text { fermés marquant les accents toniques puis } \\
\text { ouvrant les mains vers l'avant }\end{array}$ \\
\hline 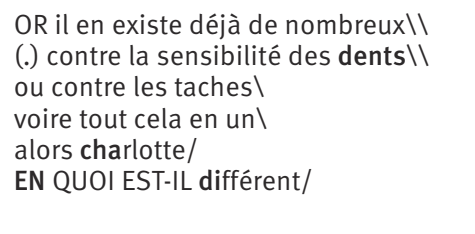 & $\begin{array}{l}\text { - mains ouvertes, bras ouverts } \\
\text { - mouvement des mains vers la droite } \\
\text { - mouvement des mains vers la gauche } \\
\text { - main droite en pince } \\
\text { - se tournant vers CD } \\
\text { - changement de plan : ML de dos, CD de } \\
\text { face, tous deux mains ouvertes }\end{array}$ \\
\hline $\begin{array}{l}\text { CD: la formule unique/ (.) } \\
\text { d'oral B } \\
\text { pro-expert/ (.) }\end{array}$ & $\begin{array}{l}\text { - poings ramenés l'un contre l'autre } \\
\text { Inscrit en bas à gauche, en surimpression } \\
\text { "Charlotte Dumézy, expert Oral-B " }\end{array}$ \\
\hline $\begin{array}{l}\text { permet d'optimiser/ (.) } \\
\text { la protection des dents / (.) } \\
\text { sur l'ENSEMBLE de ces domaines } \backslash \backslash\end{array}$ & $\begin{array}{l}\text { - mains ouvertes (coupées par le cadre) } \\
\text { - montre le mur écran main droite }\end{array}$ \\
\hline $\begin{array}{l}\text { ML: alors peut-on illustrer / son } \\
\text { action// sur la PRÉVENTION des } \\
\text { taches/ qui s'accumulent au fil du } \\
\text { temps/ par exemplel }\end{array}$ & $\begin{array}{l}\text { - montre le mur écran main gauche } \\
\text { - pointe du doigt le mot sur la liste }\end{array}$ \\
\hline$C D$ : oui venez voir & $\begin{array}{l}\text { - CD fait un geste de la main et ils vont au } \\
\text { bout de la paillasse }\end{array}$ \\
\hline \begin{tabular}{l} 
voici des languettes de composition \\
SIMILAIRE à l'émail\\
j'en plonge \\
une dans une solution du dentifrice \\
ORAL B pro-expert/ et l'autre dans \\
une solution de dentifrice \\
classique \je les rince et je les \\
mets dans du thé pendant 3 minutes\} $\\
{\text { observez l'résultat\\
} }\end{array}} &{\begin{array}{l}\text { - très gros plan sur les languettes au-dessus } \\
\text { de } 2 \text { béchers gradués contenant des liquides } \\
\text { bleu et blanc (en surimpression : Oral-B Pro- } \\
\text { Expert, Dentifrice classique) } \\
\text { - les languettes sont plongées dans un } \\
\text { bécher contenant un liquide brun } \\
\text { - Effet de montage (balayage) signalant } \\
\text { l'ellipse temporelle }\end{array}} \\
{\hline \text { ML : oh c'est^IMpressionnant\\
}} &{\begin{array}{l}\text { - Très gros plan sur les languettes qu'on sort } \\
\text { du bécher contenant du thé (ML hors-champ) }\end{array}} \\
$\hline
\end{tabular}
\end{tabular}


gestuelle emphatique ${ }^{13}$ accompagne une prononciation tonique (occlusives « explosives », /r/ vibrants, courbes mélodiques marquées, multiplication des accents d'emphase ou accents initiaux - voir de Mareüil et al., 2012) et un style langagier soutenu (notable dans la prononciation des liaisons facultatives et la multiplication des articulateurs logiques : or, voire, alors, par exemple). Quant à l'experte, on définira son style en termes d'énergie soutenue (entre les niveaux de voix neutre et soutenue) : articulation nette, mélodies intonatives marquées, lexique soutenu voire technique (plonge et non pas met, solution et non pas liquide, observez et non pas regardez) qui accompagne une gestuelle marquée. S'il y a peu de doute que son discours constitue une parole d'autorité (il s'agit d'une référence, aussi bien pour Mac Lessgy que pour le public), on constate toutefois que l'énergie qu'elle fournit dans son discours est moins élevée que celle du présentateur. De plus, si elle fait autant de gestes que ce dernier, ils sont souvent coupés par le cadre. Notons aussi que cette parole d'autorité n'est qu'un accessoire dans la boite à outils du scientifique qui contient aussi costumes (la blouse blanche) et ustensiles (dont le bécher est, tout comme l'éprouvette, un des emblèmes). Par ailleurs, son discours s'appuie sur une démonstration scientifique (dans le sens de monstration car le but de l'expérience est surtout de montrer le [non-] changement de couleur des languettes) dont elle ne tire pas elle-même de conclusions, en laissant ainsi la tâche aux téléspectateurs, et se garde alors de recommandations personnelles.

\section{La publicité Calgon sur France 5}

Sur un plateau de télévision au centre duquel se trouve une table en forme de résistance de lave-linge, le journaliste Stéphane Thebaut (ST dans les transcriptions) anime une émission factice intitulée « $\mathrm{CaCO}_{3}$ ». La publicité est construite autour de cinq épisodes ${ }^{14}$ dans lesquels le journaliste s'entretient des avantages du produit anticalcaire avec cinq invités différents (un hydrologue, un réparateur, deux chercheurs Calgon et une mère de famille) et dont nous transcrivons ici trois épisodes.

Notons d'ores et déjà que dans ce premier volet de la publicité Calgon, les styles discursifs du présentateur et de l'expert scientifique présentent des

13. Ce sont essentiellement des gestes déictiques (en particulier pour montrer et pointer) et des battements (McNeill, 1992, 2000 ; Tellier, 2010 ; Tellier et al., 2011, p. 49) : ils rythment le propos mais n'ont pas véritablement de contenu sémantique (il y aurait toutefois davantage à dire sur le rôle de la main en «pince » dans la performance argumentative). D’autres gestes sont iconiques et souvent, il y a une grande redondance entre gestes et parole (voir, par exemple, le geste de Charlotte Dumézy accompagnant son «venez voir»), ce qui contribue au style dynamique et ludique propre au genre de l'émission de vulgarisation scientifique grand public.

14. http://www.calgon.fr/programme-caco3.php, consulté en février 2014. Nous avons noté que ces publicités avaient depuis été remplacées par d'autres spots publicitaires suivant un principe de mise en scène très similaire et dans lesquels Stéphane Thebaut s'entretient cette fois avec deux réparateurs, une consommatrice, un microbiologiste, une chercheuse Calgon et une mère de famille. 
Tableau 2. Publicité $\mathrm{CaCO}_{3}$ (1) : «Les risques du calcaire»

\begin{tabular}{|c|c|}
\hline AUDIO & VISUEL \\
\hline $\begin{array}{l}\text { ST : BONJOUR } \backslash \text { et bienvenue sur le } \\
\text { plateau de } \mathrm{CaCO}_{3} \backslash \backslash(0.5)\end{array}$ & $\begin{array}{l}\text { - plan d'ensemble du plateau : ST assis, } \\
\text { main gauche tendue vers la caméra puis } \\
\text { ramenée vers le corps puis ouverte de } \\
\text { nouveau }\end{array}$ \\
\hline $\begin{array}{l}\text { emmanuel grandin vous êtes } \\
\text { |HYDROLOGUE// (0.5) } 80 \% \text { de l'eau du } \\
\text { robinet en France est donc } \\
\text { calcai ::re/ }\end{array}$ & $\begin{array}{l}\text { - changement de plan (plan taille): } \\
\text { Emmanuel Grandin de dos, ST de face, } \\
\text { main droite pince }\end{array}$ \\
\hline $\begin{array}{l}\text { HYD : oui stéphane avec certaines } \\
\text { zones où l'eau est^extrêmement } \\
\text { calcairel }\end{array}$ & $\begin{array}{l}\text { - plan taille (HYD) puis plan d'ensemble: } \\
\text { mains jointes posées sur la table (pouces } \\
\text { tendus) rythment le propos }\end{array}$ \\
\hline $\begin{array}{l}\text { ST : et comment savoir si c'est le } \\
\text { cas chez soi/ }\end{array}$ & $\begin{array}{l}\text { écran : carte de France } \\
\text { (Dureté de l'eau en France) }\end{array}$ \\
\hline $\begin{array}{l}\text { HYD : alors le calcaire dans l'eau } \\
\text { n'est pas visible à l'oeil nul (.) }\end{array}$ & - plan poitrine (HYD) \\
\hline $\begin{array}{l}\text { mais vous pouvez en vérifier le } \\
\text { niveau à l'aide euh d'une } \\
\text { bandelette-TEST comme celle-ci\ } \\
\text { voilà il vous suffit de tremper la } \\
\text { bandelette juste une secondel et au } \\
\text { bout d'une minute le résultat précis } \\
\text { apparait } \backslash \text { ben dans ce cas précis } \\
\text { elle devient ROUGE ça veut dire } \\
\text { que cette eau est TRÉS calcairell }\end{array}$ & $\begin{array}{l}\text { - plan taille (HYD) + ST en amorce } \\
\text { - HYD montre la bandelette à ST } \\
\text { - gros plan : il la plonge dans un bécher } \\
\text { d'eau posé sur la table } \\
\text { écran : bandelettes eau douce/eau dure } \\
\text { - plan taille : ST de face regarde la bande- } \\
\text { lette (HYD en amorce) } \\
\text { - gros plan sur la bandelette tenue en } \\
\text { main }\end{array}$ \\
\hline $\begin{array}{l}\text { ST : et alors quelles sont les } \\
\text { conséquences/ }\end{array}$ & $\begin{array}{l}\text { - plan taille : ST de face (HYD en amorce) } \\
\text { - mouvement de la main }\end{array}$ \\
\hline $\begin{array}{l}\text { HYD : pour nous stéphane c'est sans } \\
\text { danger \ mais pour vos appareils } \\
\text { électroménagers/(.) c'est^une autre } \\
\text { affaire } \backslash \text { regardez l’EFFET sur cette } \\
\text { bouilloi ::re }\end{array}$ & $\begin{array}{l}\text { - plan taille (HYD) : souligne le propos } \\
\text { d'un hochement de la tête } \\
\text { - sourit, hausse les sourcils } \\
\text { - cliché : résistance de bouilloire couverte } \\
\text { de calcaire (zoom avant) }\end{array}$ \\
\hline \multicolumn{2}{|l|}{ ST : ah oui effectivement $\backslash \backslash$} \\
\hline $\begin{array}{l}\text { HYD : alors imaginez votre lave- } \\
\text { linge \le calcaire se dépose (.) sur } \\
\text { la résistance } \backslash \text { ce qui peut mener à } \\
\text { des dysfonctionnements } \backslash \text { (.) et dans } \\
\text { certains cas/jusqu’à la pa ::nnel】 }\end{array}$ & $\begin{array}{l}\text { - cliché : résistance de lave-linge couverte } \\
\text { de calcaire (zoom avant) } \\
\text { - Plan taille (HYD) : mains jointes, pouces } \\
\text { l'un contre l'autre, marque le propos }\end{array}$ \\
\hline $\begin{array}{l}\text { ST : vous l'aurez compris vos } \\
\text { machines à laver sont^}{ }^{\wedge} \text { EN DANGER } \backslash\end{array}$ & $\begin{array}{l}\text { - Plan taille ST (regard caméra), main } \\
\text { pince }\end{array}$ \\
\hline
\end{tabular}


ressemblances avec ceux que nous avons analysés dans le spot Oral-B. Le style discursif de l'hydrologue se caractérise par une articulation nette (entre les voix neutre et soutenue), un lexique technique et une organisation discursive précise (alors, voilà, ça veut dire) construisant un raisonnement. Le contrôle articulatoire accompagne la maitrise intellectuelle liée à l'argumentation : le ton est résolument didactique. Tout comme l'experte Oral-B, il fait une démonstration qui s'appuie sur une série de preuves présentées sur le plateau (bandelettestest, photos, etc.) mais ne fait pas de recommandation. Le style du présentateur est moins énergique que celui de Mac Lessgy et ne présente que quelques pointes vers un niveau de voix soutenu (en début de scène). Ceci s'explique entre autres par une tendance à s'aligner sur le discours de son interlocuteur (tendance confirmée dans les épisodes suivants). Ainsi, Kerbrat-Orecchioni (1987, p. 324) note la complexité du statut de celui qui pose les questions:

S'il [l'intervieweur] est bien en position haute dans la mesure où il «mène» l'interaction, oriente le débat et prend la plupart des «initiatives», il abdique sur un autre front puisque son rôle est moins de parler que de susciter la parole de l'autre, auquel il laisse le soin de fournir l'essentiel de la matière conversationnelle.

Finalement, on remarque qu'une mise en scène et un montage rythmés atténuent l'effet de lourdeur dû à l'abondance de texte (peu fréquent dans les publicités où la norme est souvent à une narration brève, en voix off).

$\mathrm{Si}$, tout comme l'experte Oral-B et l'hydrologue, le réparateur se prête au jeu de la démonstration sur le plateau, remarquons que celle-ci s'appuie sur des conséquences (eau stagnante et filtre) plutôt que sur un test scientifique. Le visuel apporte une dimension redondante : ce qui est dit est aussitôt montré à l'aide de preuves matérielles ou photographiques (soulignant l'expérience du réparateur car il a lui-même prélevé cette eau stagnante et retiré ce filtre). Étant donné l'acception du terme «expert » telle que donnée par le Petit Robert («personne choisie pour ses connaissances techniques et chargée de faire, en vue de la solution d'un procès, des examens, constatations ou appréciations de fait»), on peut confirmer le statut d'expert du réparateur qui non seulement apprécie des faits (états des machines) et propose une solution (utiliser un produit contre le calcaire), mais fait ainsi une recommandation. Par ailleurs, si le niveau lexical reste technique (résidus, eau stagnante, évaluer les dégâts, prélever), on note des différences sur les plans syntaxique (phrases simples entrecoupées de pauses et d'hésitations) et phonétique (articulation moins nette, courbes mélodiques peu marquées, voix douce). Tous ces éléments ont été soigneusement scénarisés et mis en scène pour créer un effet d'authenticité. Cette authenticité toute factice est problématique du point de vue de la déontologie publicitaire. Ainsi, en Belgique, à la suite d'une enquête menée par la RTBF et alors que les prétendus experts s'avérèrent n'être que des comédiens, le JEP (Jury d'éthique publicitaire) demanda le retrait de la publicité. Retraçant 
Tableau 3. Publicité $\mathrm{CaCO}_{3}$ (2) : «La menace des résidus »

\begin{tabular}{|c|c|}
\hline AUDIO & VISUEL \\
\hline $\begin{array}{l}\mathrm{ST}: \text { BONJOUR } \backslash \text { et bienvenue sur le } \\
\text { plateau de } \mathrm{CaCO}_{3} \backslash \backslash(0.5)\end{array}$ & $\begin{array}{l}\text { - vue d'ensemble A : ST assis, main } \\
\text { gauche tendue vers la caméra, rame- } \\
\text { née vers le corps puis ouverte, } \\
\text { main pince puis index pointé caméra }\end{array}$ \\
\hline $\begin{array}{l}\text { hier nous avons vu que le CALCAI ::RE } \\
\text { est un ennemi pour votre lave-lin ::ge } \backslash\end{array}$ & $\begin{array}{l}\text { - plan B poitrine (ST) se tournant vers } \\
\text { REP }\end{array}$ \\
\hline $\begin{array}{l}\text { josé varenne vous êtes réparateur/ } \\
\text { y a-t-il d'autres dangers pour nos machines/ }\end{array}$ & $\begin{array}{l}\text { - plan C taille (ST de face, REP de } \\
\text { dos) : index pointé vers REP }\end{array}$ \\
\hline 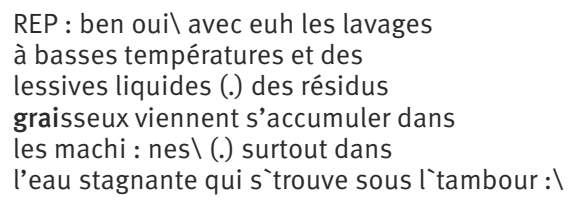 & $\begin{array}{l}\text { - plan D poitrine (REP) : geste coupé } \\
\text { par le cadre (peu visible) } \\
\text { - retour plan C : ST de face attentif } \\
\text { - plan D : REP poings collés } \\
\text { - plan A : écran au fond projetant un } \\
\text { extrait de film }\end{array}$ \\
\hline ST : comment savoir si c'est grave/ & - main de ST tendue vers REP \\
\hline $\begin{array}{l}\text { REP : alors moi pour évaluer les } \\
\text { dégâts/je PRÉLEEVE cette eau (1) } \\
\text { d'ailleurs en voici justement un échantillon :। }\end{array}$ & $\begin{array}{l}\text { - extrait (plein écran) : deux gros plans } \\
\text { sur une main tenant un bocal dans } \\
\text { lequel se déverse de l'eau marron (vue } \\
\text { de profil et du dessus) } \\
\text { - retour plateau plan A : REP donne } \\
\text { le bocal }\end{array}$ \\
\hline ST : oui :::\ & $\begin{array}{l}\text { - gros plan sur le bocal qui change de } \\
\text { mains }\end{array}$ \\
\hline $\begin{array}{l}\text { REP : en fait c'est comme si vous } \\
\text { laviez votre linge avec euh de l'eau sa :::le\l }\end{array}$ & $\begin{array}{l}\text { - plan E : REP de face hausse les } \\
\text { sourcils et soupire, ST ouvre le bocal et } \\
\text { sent l'eau }\end{array}$ \\
\hline $\begin{array}{l}\text { ST : ouf et ça sent pas très bon en } \\
\text { plus HEIN/(.) mais dites-moi\à la } \\
\text { maison\ on n neut pas démonter sa machine// }\end{array}$ & $\begin{array}{l}\text { - plan C : ST grimace et referme le } \\
\text { bocal } \\
\text { - plan F très rapproché (ST) } \\
\text { - plan E : ST main ouverte vers REP } \\
\text { REP geste des mains }\end{array}$ \\
\hline 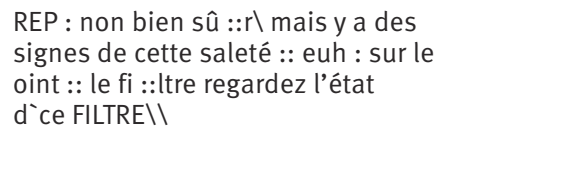 & $\begin{array}{l}\text { - extrait : gros plan d'une main } \\
\text { passant sur un joint et relevant une } \\
\text { substance brune } \\
\text { - plan E : REP prend un filtre posé } \\
\text { dans un récipient sur la table }\end{array}$ \\
\hline ST : ah ouil\ & $\begin{array}{l}\text { - gros plan du filtre dans la main } \\
\text { - Plan F: ST acquiesce très attentif }\end{array}$ \\
\hline $\begin{array}{l}\text { REP : la seule solution pour moi } \\
\text { c'est d'utiliser un produit/CONTRE } \\
\text { le calcaire et contre la formation d'résidus } \backslash \backslash\end{array}$ & $\begin{array}{l}\text { - Plan E : REP regarde le filtre et le } \\
\text { désigne de la main }\end{array}$ \\
\hline
\end{tabular}


les événements sur le site de la chaine belge, le journaliste présente en ces termes les arguments préludant à la décision du jury :

Si l'utilisation de faux experts n'est pas strictement interdite en publicité, les noms et fonctions mentionnés doivent, eux, réellement exister. Ce spot va en outre un cran plus loin dans la manipulation, en mélangeant un vrai journaliste à des personnages fictifs. (RTBF 2013 [nous soulignons])

Une confusion des genres qui abuse alors de la confiance des consommateurs au sens de l'article 3 du Code ICC15. Le prochain volet confirme cette tendance à imiter le réel et à brouiller les pistes.

Cet autre volet de la publicité présente un changement notable de style discursif, en particulier pour l'invitée : douceur des contours articulatoires et intonatifs (niveau de voix neutre voire minimale), juxtaposition des phrases avec peu, voire sans mot de liaison (proche de la parataxe), lexique simple. Le texte est brut et présente des défauts (pauses, hésitations) car il doit donner l'impression qu'il n’a pas été répété. Tout ceci a ainsi pour but de créer un sentiment d'authenticité, de proximité et de familiarité (renforcé sur le plan visuel par un surcroit d'expressions faciales incarnant la sympathie et la complicité des interlocuteurs). Ainsi Bénédicte fait-elle autorité car son discours est marqué du sceau de la crédibilité et de la véracité. Notons aussi que ce volet est le premier à inclure le prénom de l'invitée dans son titre. À la lecture du texte montré ensuite lors de son témoignage ("Un foyer français », etc.), on comprend qu'elle incarne un échantillon représentatif de la population française : la ménagère de moins de 50 ans. Rappelant que, selon Oscar Wilde, "un expert, c'est un homme ordinaire qui donne son avis... quand il n'est pas à la maison », on argumentera que, dans les affaires du foyer, la mère de famille est la référence, et donc l'experte. C'est aussi une figure archétypique de la publicité de lessive. Toutefois, Bénédicte ne fait pas de démonstration (c'est Stéphane Thebaut qui, à la suite de son témoignage, présente des clichés pris au microscope) ni de recommandation (même si elle déclare par deux fois utiliser le produit). Considérant l'acception communément admise du terme «autorité » et telle qu'elle est donnée par le Larousse («crédit, influence, pouvoir dont jouit quelqu'un ou un groupe dans le domaine de la connaissance ou d'une activité quelconque, du fait de sa valeur, de son expérience, de sa position dans la société, etc.» [nous soulignons]), cet exercice narratif est assez pour convaincre les téléspectateurs. La prestation de Bénédicte serait sans doute moins convaincante si ces derniers étaient clairement informés du fait que celle qui témoigne en tant que mère de famille est une actrice qui est peut-être célibataire et sans enfant. Ainsi, comme le rappelle un journaliste :

15. «La communication commerciale doit être conçue de manière à ne pas abuser de la confiance des consommateurs ou à ne pas exploiter le manque d'expérience ou de connaissance des consommateurs.» (2011, p. 7) 
Tableau 4. Publicité $\mathrm{CaCO}_{3}$ (3) : «Le témoignage de Bénédicte»

\begin{tabular}{|c|c|}
\hline AUDIO & VISUEL \\
\hline 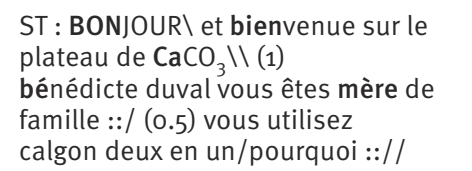 & $\begin{array}{l}\text { - plan d'ensemble A : ST assis, main gauche } \\
\text { tendue vers la caméra } \\
\text { - plan B poitrine (ST) se tournant vers BD } \\
\text { - plan C taille (ST de face, BD de dos) : mains } \\
\text { ouvertes vers le ciel }\end{array}$ \\
\hline $\begin{array}{l}\text { BD : (.) j’ai } 3 \text { enfants :/ (0.5) } \\
\text { je fais des machines tout l'temps/(1) } \\
\text { pour moi c'est important d'pouvoir } \\
\text { compter sur mon lave-lin : ge\(1) } \\
\text { donc je l’protège en utilisant } \\
\text { calgon à chaque lava: gel\ }\end{array}$ & $\begin{array}{l}\text { - plan D poitrine }(\mathrm{BD}) \text { : yeux au ciel (= expri- } \\
\text { mant la réflexion), mains jointes sur la table } \\
- \text { écran } 1: \text { "Un foyer français " } \\
- \text { écran } 2: \text { "Un foyer français fait en moyenne } \\
250 \text { machines par an - Source TNS Infratest } \\
-2008 \text { " }\end{array}$ \\
\hline ST : vous craignez la panne// & $\begin{array}{l}\text { - plan C taille (ST de face, BD de dos) : mains } \\
\text { jointe sur la table }\end{array}$ \\
\hline $\begin{array}{l}\text { BD : (1) j’irai pas jusque-là mais } \\
\text { je préfère ne pas prendre de } \\
\text { risques } \backslash(0.5) \text { vous m'imaginez aller } \\
\text { à la laverie/(o.5) ou pire/faire } \\
\text { mes lessives chez mes parents// }\end{array}$ & $\begin{array}{l}\text { - plan D poitrine }(\mathrm{BD}) \text { : moue } \\
\text { - plan } C \text { taille }(\mathrm{ST} \text { de face, } \mathrm{BD} \text { de dos) : sourit } \\
\text { et acquiesce } \\
\text { - plan } \mathrm{E}: \mathrm{ST} \text { et } \mathrm{BD} \text { de profil face à face }\end{array}$ \\
\hline ST : ouh làl\ & $\begin{array}{l}\text { - plan C taille (ST de face, BD de dos) : une } \\
\text { main levée au ciel }\end{array}$ \\
\hline $\begin{array}{l}\mathrm{BD}: \text { mais c`n'est pas tout } \backslash \text { depuis } \\
\text { qu 'j'utilise Calgon deux en un :/ (.) } \\
\text { i`m`semb`que mon linge est moins } \\
\text { rêche\(.) plus doux }\end{array}$ & $\begin{array}{l}\text { - plan D poitrine (BD) : sourire, } \\
\text { moue, yeux au ciel, puis hoche la tête }\end{array}$ \\
\hline $\begin{array}{l}\mathrm{ST} \text { : vous avez raison } \backslash \text { regardez ces } \\
\text { images prises au microsco ::pe }\end{array}$ & $\begin{array}{l}\text { - plan C taille (ST de face, BD de dos) : montre } \\
\text { BD puis l'écran de l'index }\end{array}$ \\
\hline
\end{tabular}


Au cours de sa carrière, un acteur professionnel peut ainsi interpréter un capitaine de chalutier pour une marque de thon en conserve, un nutritionniste pour une marque de yaourt, un réparateur de machine à laver ou encore un scientifique pour une marque de produit anticalcaire. Le profil des acteurs est sélectionné sur des questions de parts de marché, et en fonction du consommateur visé par le message. Les agences de publicité écrivent des scénarios, qui sont validés par leurs clients. Ces derniers contactent ensuite des agences de comédien pour un casting, et si un candidat correspond à ce que la marque recherche, il est engagé. Bien qu'il ne soit pas un expert comme voudrait le faire croire la publicité, le message qu'il délivre n'est en théorie pas mensonger : le message est travaillé en amont par la production, qui ne peut faire dire n'importe quoi à ses acteurs. (De Morel, 2012 [nous soulignons])

Si les publicitaires ont recours à des acteurs pour jouer ces divers rôles, leur souhait est néanmoins que ceux-ci sonnent juste (qu'ils semblent authentiques). Ainsi, il leur est parfois demandé d'être maladroits, voire de jouer faux. Une tendance confirmée par l'utilisation de personnalités présentant leurs propres produits (coiffeurs-hommes d'affaire) ou d'autres (sportifs clamant les mérites de jus de fruit ou de parfums) : un phénomène connu en anglais sous le nom d'endorsement (Biswas et al., 2006) ${ }^{16}$. Si la pratique est fréquente de l'autre côté de l'Atlantique, la mise en scène à la française reste une exception culturelle : en effet, on aurait raison de se demander, tout comme ce journaliste de L'Express, "Pourquoi s'acharner à mettre en scène des sportifs qui jouent comme des pieds ? "17 Nous postulons que cette maladresse est mise en scène comme une garantie d'authenticité dans laquelle il faut entendre : ces gens n'ont pas suffisamment d'éloquence pour mentir (point sur lequel nous reviendrons plus tard). Nous sommes alors proches du dire-vrai : un discours qui engage celui et celle qui le prononce. C'est le cas de Pierre Papillaud, président de l'entreprise Rozana, lorsqu'il vante les mérites de son eau gazeuse dans une publicité qui illustre parfaitement cette esthétique de l'authentique qui multiplie maladresses de jeu et faux raccords ${ }^{18}$. Remarquons que la toute dernière publicité de la même marque se joue aussi de la véracité des discours publicitaire et politique.

16. En français, on parle d'endossement : «L'endossement publicitaire consiste à demander à une personnalité connue, généralement une célébrité, de s'approprier explicitement le discours promotionnel de la marque.» (Viot, 2012)

17. http://www.lexpress.fr/styles/saveurs/la-pub-pour-l-agro-alimentaire-recrute-meme-les-sportifs_1235374.html.

18. http://www.youtube.com/watch?v=Cxtw3EAyZfl. 
La publicité Rozana (2013)

Tableau 5. La publicité Rozana (2013)

\begin{tabular}{|c|c|}
\hline AUDIO & VIDÉO \\
\hline 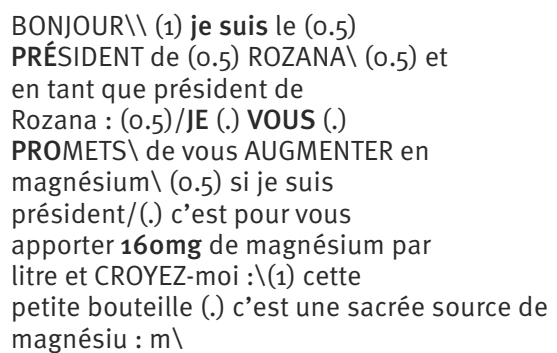 & $\begin{array}{l}\text { - plan taille de Pierre Papillaud, au podium, } \\
\text { derrière le micro (caméra face) } \\
\text { - plan moyen (en pied) caméra droite } \\
\text { - plan taille caméra face, main pince } \\
\\
\text { PP balaie son public du regard } \\
\text { - gros plan sur la bouteille d'eau } \\
\text { - plan moyen caméra droite } \\
\text { - plan taille caméra face } \\
\text { PP regarde le public à droite }\end{array}$ \\
\hline
\end{tabular}

Sur le plan technique, la mise en scène participe à l'esthétique de l'authentique déjà mise en place par les maladresses de jeu de Pierre Papillaud. Le montage haché et les sautes de plans renforcent un effet de discours qui aurait été filmé sur le vif et non pas mis en scène ${ }^{19}$. Cette parodie du discours politique est paradoxale puisqu'en imitant le politicien et en invoquant par là même un imaginaire populaire d'homme politique qui ne tient que rarement ses promesses, Pierre Papillaud semble saper son propre message. Toutefois, pour Marc Bonhomme, «La parodie [du politique] s'inscrit dans un régime ludique basé sur le détachement humoristique» (2012, p. 51). Les discours publicitaires recyclent et reproduisent des genres discursifs qui participent à des mises en scène d'archétypes (l'expert, la mère de famille, l'homme politique, etc.) déjà connus des téléspectateurs et s'appuient ainsi sur un savoir partagé. Dans un article intitulé "Styling the other», Ben Rampton rappelle «the dense interpenetration of local performance with styles of speech that are reflexively designed, produced and disseminated through mass institutional and/or electronic communication systems, whether mainstream or counter-cultural» (1999, p.423).

\section{Proposition d'interprétation : imaginaire de la parole d'expertise}

La figure 1 montre une mise en champ des voix d'experts analysées dans les publicités selon les axes $x$ (relâché $\rightarrow$ énergique) / (maladroit $\rightarrow$ éloquent) et $y$ (authentique $\rightarrow$ mis en scène). Nous avons inscrit sur ces axes les variations de niveaux phonatoires (de la parole maitrisée et énergique des scientifiques à

19. http://www.youtube.com/watch?v=_IIWLubbJNU. 
la parole maladroite et authentique des pros), tout en tenant compte des ambiguïtés qui peuvent exister pour certaines voix (par exemple, les présentateurs jouent leur propre rôle mais ces rôles sont des persona médiatiques qui sont mises en scène).

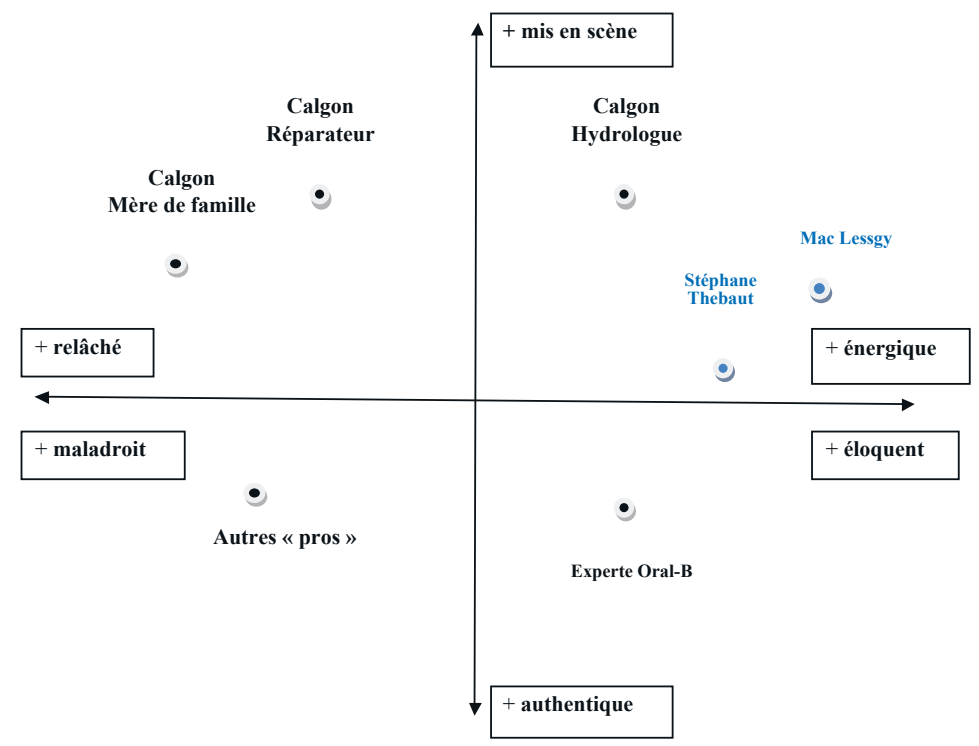

Figure 1. Les mises en scène des voix d'experts

En ce qui concerne la variété des voix d'experts trouvée dans les publicités, Dipayan Biswas, Abhijit Biswas et Neel Das (2006) soulignent la nécessité de distinguer l'endossement publicitaire par une célébrité de celui d'un expert à proprement parler. Leurs travaux montrent ainsi que le crédit apporté à l'expertise de l'endosseur dépend du degré de technicité du produit. Ainsi, dans le cas d'un médicament, une confiance plus importante est accordée au médecin ou à un scientifique. Selon eux (2006, p. 19), "[e]xpertise is topic-specific; an expert source must possess expertise on a particular topic rather than having it at the generalized level ». Qu'en est-il alors de l'endossement de produits aussi anodins que du dentifrice ou des produits d'entretien? On postulera que les publicitaires auront intérêt à manier une diversité de voix d'expertise car, comme le précise Alain Anquetil (2012) :

Alors que le processus psychologique sous-jacent à un endossement par une célébrité est un processus d'identification, le processus sous-jacent à un endossement par un expert est une internalisation, i.e. l'acceptation par le sujet d'une influence qui est «cohérente avec son système de valeurs et qui s'avère utile pour résoudre un problème particulier». 
C'est dans le premier type de processus que se range par exemple la voix de Bénédicte, dont l'expertise repose sur la capacité à fournir des informations et des recommandations en fonction de son expérience (Biswas et al., 2006). Le crédit donné à cet endossement est alors fonction du degré d'authenticité accordé à l'expérience.

La demande de retrait de la publicité Calgon par le Jury d'éthique publicitaire belge souligne ainsi le potentiel décalage qui existe entre la réelle mise en scène des voix d'experts (jouées par des acteurs/actrices) et la perception par les téléspectateurs de ces voix comme authentiques ${ }^{20}$. On comprend mieux aussi l'avantage des publicitaires à conserver une forme d'ambiguïté dans l'identification des participants (acteurs présentés comme de vraies personnes / experts). En effet, s'il est évident que la perception de la parole d'autorité dépend du type d'expertise de l'endosseur (scientifique ou basée sur l'expérience), celle-ci semble aussi fonction de la faculté des publicitaires à mettre en scène, par le biais d'outils dramatiques (tels que l'énergie phonatoire ou le degré d'articulation), une parole d'expérience qui sera entendue comme authentique.

Par ailleurs, nous aimerions souligner qu'aucune des voix d'experts analysées pour cette étude ne présente d'accents notables (sociaux ou régionaux). Nous avons alors affaire à ce que Samantha Warhurst, Patricia McCabe et Catherine Madill (2013), dans leur étude des voix médiatiques idéales, appellent des voix «sans faute » : une caractéristique qui fait ainsi émerger un autre aspect idéologique de la parole d'autorité, une forme d'hygiène verbale (Cameron, 1994, 1995) qui a pu trouver dans le passé, et en particulier sur la scène politique, des échos notables.

\section{Du dire-vrai au parler-vrai en politique}

Gardant à l'esprit la ligne éditoriale de Mots. Les langages du politique, nous ouvrons ici une parenthèse où nous observons en quoi nos analyses de la parole d'autorité dans la publicité, mais aussi des mises en scène du discours authentique dans les médias, peuvent éclairer les études des discours politiques, dont celles du parler-vrai, en permettant de repenser le concept d'authenticité ou de naturel comme des construits idéologiques et culturels.

Si la formule avait été inaugurée dans un livre de Michel Rocard publié en $1979^{21}$ puis remise au goût du jour par les phrases chocs du tout d'abord

20. En réponse à cette injonction, les changements récents sur le site de Calgon semblent cette fois présenter de vrais gens. Constatons toutefois que l'ambiguïté demeure et que si les publicités déclarent avoir été filmées «chez des particuliers », on ne peut jamais être certain de l'authenticité des professions et des occupations indiquées en surimpression.

21. Pour plus de détails sur l'historique de cette formule, voir Tournier (1990, p. 197). 
ministre de l'Intérieur puis président Nicolas Sarkozy, le « parler-vrai » connait un récent retour en force dans les médias. Cet article récent du journal Le Monde s'ouvrait sur ces mots : "Concis, musclé : il y avait du “parlervrai” dans la déclaration de politique générale de Manuel Valls, mardi 8 avril, à l'Assemblée. »22 S'opposant à la langue de bois des politiciens et aux discours hermétiques des technocrates ${ }^{23}$, la formule désigne avant tout un contenu, le but de l'exercice étant de dire la vérité et de parler concret. Toutefois, constatons que, de la même manière que le dire-vrai analysé dans nos publicités, "parler vrai» est aussi adopter un genre discursif codé, dont l'aspect authentique est loin d’être improvisé ou gratuit :

Aujourd'hui, le relâchement du langage devient un outil de communication, preuve de «naturel» et de proximité avec les «vrais gens». Le parler-vrai donne l'impression de rompre avec la langue de bois, et l'obscur langage technocrate, l'«énalangue» comme la nomme Christian Delporte.

Le parler-vrai c'est le parler «comme tout le monde». C'est dire je parle comme vous - je suis comme vous - vous pouvez me faire confiance. Le parler-vrai ce n'est pas chercher à dire la vérité. C'est parler avec des mots "vrais », que tout le monde comprend. S’identifier aux Français. ${ }^{24}$

Comme dans les discours des vrais gens des publicités que nous avons analysées, cette langue produite pour être entendue comme venant du cœur n'échappe pas au contrôle de son locuteur. Elle fait partie d'une panoplie d'outils discursifs utilisés pour instaurer confiance et sympathie et permettre l'identification. «Quand on "parle vrai”, on utilise un langage qui rend floue la frontière entre le politique et le civil » (Lemarié, 2010). De la même manière que le personnage de Bénédicte s’appuyait sur un rôle de mère de famille comme symbole d'expérience et pour mieux convaincre les téléspectateurs, Ségolène Royal ou Sarah Palin invoquaient leur statut de mère comme argument politique. Ainsi la parole d'autorité s'articule non seulement autour d'avatars narratifs qui existent dans différents sites discursifs et qui sont familiers du grand public, mais repose aussi sur un effet de naturel mis en scène et dont la fonction rhétorique et argumentative ne doit pas être négligée par le chercheur.

Dans cet article, l'analyse de deux publicités nous a permis de mettre en lumière un imaginaire de la parole d'autorité au sein duquel des variétés indexent différents types d'expertise (scientifique, professionnelle ou d'expérience). Parmi une palette de traits linguistiques, les publicitaires choisissent les caractéris-

22. http://www.lemonde.fr/politique/article/2014/04/o9/au-dela-du-parler-vrai-le-premierministre-sera-juge-sur-ses-actes_4398180_823448.html.

23. Parole publique devenue «langue morte», selon les mots de Manuel Valls dans un article du quotidien Libération (8 avril 2014) dont le titre faisait lui aussi mention du parler-vrai : http:// www.liberation.fr/politiques/2014/04/o8/manuel-valls-le-parler-vrai-selectif_994212.

24. http://tout-ca.com/2010/01/22/le-parler-vrai-la-nouvelle-langue-de-bois/. 
tiques à accentuer en fonction du genre d'expertise ou du degré de crédibilité (voire d'authenticité) qu'ils souhaitent que les téléspectateurs accordent aux experts ainsi mis en scène. En se concentrant particulièrement sur les traits d'énergie phonatoire et d'articulation, cette étude a fait émerger que, si le standard publicitaire mettait souvent en scène un niveau de voix soutenue, le niveau neutre permettait aussi d'indexer familiarité, proximité et sincérité.

Par ailleurs, le choix des publicitaires d'adopter différents types d'endossements et diverses voix d'expertise s'inscrit dans la modernité radicale telle que la définit Anthony Giddens (1991). En effet, à une époque où les scientifiques agissent moins qu'auparavant comme les gardiens des traditions et mettent moins en avant la certitude des savoirs, mais se basent davantage sur les méthodologies du doute, de la critique ou de la déconstruction (les controverses deviennent alors le mode de relation de la science à la société), la parole d'autorité est sans cesse réévaluée. L'expertise qui s'appuie sur l'expérience et les connaissances techniques ou pratiques (plutôt que sur un statut lié à un savoir toujours révisable) instaure une continuité qui rend plus fiable le discours d'autorité25.

\section{Références}

ANQUETIL Alain, 2012, "L'évaluation morale des cautions des experts dans la publicité», ESSCA. Le blog d'éthique des affaires, http://sites.essca.fr/blog/ethique/ billets/L_evaluation-morale-des-cautions-des-experts-dans-la-publicite-1509.htm (consulté le 12 mai 2014).

ARPP (Autorité de régulation professionnelle de la publicité), 2002, Allégations santé. http://www.arpp-pub.org/IMG/pdf/Allegations_sante.pdf (consulté le 12 mai 2014).

Barbet Denis, Desmarchelier Dominique, 2012, "Au croisement de deux mondes», Mots. Les langages du politique, no 98, p. 5-13.

Bıswas Dipayan, Bıswas Abhijit, DAs Neel, 2006, «The differential effects of celebrity and expert endorsements on consumer risk perceptions. The role of consumer knowledge, perceived congruency, and product technology orientation », Journal of Advertising, $\mathrm{n}^{\circ} 35$ (2), p. 17-31.

Bon номme Marc, 2012, "Quand la publicité parodie la politique», Mots. Les langages du politique, $\mathrm{n}^{\circ} 98, \mathrm{p} .31-45$.

Boula de MareüIl Philippe, Rilliard Albert, Allauzen Alexandre, 2012, «Variation diachronique dans la prosodie du style journalistique : le cas de l'accent initial», Revue française de linguistique appliquée, nº 1 (17), p. 97-111.

CAmERon Deborah, 1994, "Verbal hygiene for women. Linguistics misapplied? », Applied Linguistics, $\mathrm{n}^{0} 15, \mathrm{p} .382-398$.

25. Nous remercions Claire Oger de sa lecture et de ses commentaires sur une version préliminaire de cet article. Nous signalons que tout problème structural, erreur, ou omission reste notre seule responsabilité. 
- 1995, Verbal Hygiene, Londres, New York, Routledge.

ChAmbRe DE COMmERCE INTERNATIONALE, 2011, CODE ICC consolidé sur les pratiques de publicité et de communication commerciale, http://www.icc-france.fr/chambrede-commerce-internationale-page-3-60-190-Code_consolide_sur_la_publicite. html (consulté le 12 mai 2014).

Coffin Alice, 2013, «Pourquoi les journalistes parlent bizarrement... Affaire à suivre», 20 minutes, 25 février 2013. http://www.20minutes.fr/medias/television/1107031pourquoi-journalistes-parlent-bizarrement-affaire-a-suivre (consulté le 12 mai 2014).

GIDDENS Anthony, 1991, Modernity and self identity. Self and society in the Late Modern Age. Cambridge, Polity Press.

Kerbrat-ORReChioni Catherine, 1987, Décrire la conversation, Lyon, Presses universitaires de Lyon.

LEMARIÉ Sarah, 2010, «Le parler-vrai : la nouvelle langue de bois», Tout ça, http://tout-ca. com/2010/01/22/le-parler-vrai-la-nouvelle-langue-de-bois/(consulté le 12 mai 2014).

MCNeILl David, 1992, Hand and Mind. What Gestures Reveal about Thought, Chicago, The University of Chicago Press.

- éd., 2000, Language and Gesture, Cambridge, Cambridge University Press.

MOREL Pierrick (de), 2012, "Les experts des publicités sont-ils de vrais scientifiques? ", Slate.fr, http://www.slate.fr/story/64241/experts-publicites-vrais-scientifiques (consulté le 12 mai 2014).

PetIT Gérard, 2000, "Le statut d'expert dans la presse quotidienne », Les Carnets $d u$ Cediscor, n०6, p. 63-79.

PlanchenAult Gaëlle, 2012, "City boys and country bumpkins. What films tell us about the folk linguistics of accented varieties», communication présentée au Sociolinguistics Symposium 19, Berlin.

RAMPTON Ben, 1999, "Styling the other. Introduction», Journal of Sociolinguistics, $\mathrm{n}^{\circ} 3$ (4), p. 421-427.

RÉVIS Joana, 2013, La voix et soi. Ce que notre voix dit de nous, Bruxelles, De Boeck Solal. RTBF, Médias (Ju. VI.), 2013, «Une pub Calgon interdite suite à l'enquête d'“On n'est pas des Pigeons" », http://www.rtbf.be/info/medias/detail_une-pub-calgon-interdite-suitea-l-enquete-de-on-n-est-pas-des-pigeons?id=7988802 (consulté le 12 mai 2014).

Tellier Marion, 2010, "Les gestes des politiques», Déshabillons-les, Public Sénat, émission diffusée le 30 novembre 2010, en ligne : http://replay.publicsenat. $\mathrm{fr} / \mathrm{vod} / \mathrm{deshabillons-les/les-gestes-des-politiques/marion-tellier,philippe-}$ turcher, mariette-darrigrand/66557 (consulté le 12 mai 2014).

Tellier Marion, Guardiola Mathilde, BIGI Brigitte, 2011, «Types de gestes et utilisation de l'espace gestuel dans une description spatiale. Méthodologie de l'annotation », Actes, Atelier DEGELS, $18^{\mathrm{e}}$ conférence annuelle Traitement automatique des langues naturelles (TALN), 27 juin - 1 er juillet 2011, Montpellier, Université Montpellier 2, p. 45-56.

Tournier Maurice, 1990, «Le parler vrai, ou qu'est-ce qu'un néologisme», Mots, n²2, p. 97-100 (texte repris dans Propos d'étymologie sociale, t. II, Des mots en politique, sous le titre : «Le parler vrai, néologisme politique», Lyon, ENS Éditions, 2002, p. 193-196).

TRÉPOS Jean-Yves, 1996, Sociologie de l'expertise, Paris, Presses universitaires de France. 
Vanoye Francis, Goliot-LÉté Anne, 1992, Précis d'analyse filmique, Paris, Nathan Université.

Vıот Catherine, 2012, «Endossement, pseudo-endossement et co-endossement d'une marque patronymique. Potentiel et intérêt pour une stratégie marketing », Décisions marketing, nº66, p. 21-33.

WARHURST Samantha, MCCABE Patricia et MADILL Catherine, 2013, "What makes a good voice for radio. Perceptions of radio employers and educators», Journal of Voice, $\mathrm{n}^{0} 27$ (2), p. 217-224.

Wolton Dominique, 1995, "Le savant, l'expert, le politique». Libération, http:// www.liberation.fr/tribune/1995/01/12/le-savant-l-expert-le-politique_120189 (consulté le 12 mai 2014). 


\section{Annexe}

\section{Convention de transcription (d'après la Convention ICOR)}

\begin{tabular}{|c|c|}
\hline Convention & Symboles utilisés \\
\hline Élision standard & ' (Apostrophe) \\
\hline $\begin{array}{l}\text { Élision non standard (absence ou non } \\
\text { prononciation d'un ou plusieurs sons) }\end{array}$ & - (Antiquote) \\
\hline Liaison facultative prononcée & «^» entre les deux mots liés \\
\hline Allongement & $\begin{array}{l}\text { Son allongé noté par des :::: (nombre en fonc- } \\
\text { tion de la longueur de l'allongement) }\end{array}$ \\
\hline Micro pause et pauses & $\begin{array}{l}\text { Pauses d'une durée inférieure à o, } 2 \mathrm{~S} \text {. notées } \\
\text { par (.); d'une durée supérieure à } 0.2 \mathrm{~S} \text {. notées } \\
\text { par }(0.5) \text { pour } 1 / 2 \text { seconde, (1) pour } 1 \text { seconde, } \\
\text { etc. }\end{array}$ \\
\hline Montée et chute intonative & $\begin{array}{l}\text { Montées et chutes intonatives notées respecti- } \\
\text { vement par «/» et «\» }\end{array}$ \\
\hline Saillance perceptuelle & $\begin{array}{l}\text { Segments caractérisés par une saillance } \\
\text { perceptuelle (intensité accrue ou autre) notés } \\
\text { en majuscules }\end{array}$ \\
\hline Accent initial & $\begin{array}{l}\text { Segments caractérisés par un accent initial } \\
\text { indiqués par des caractères gras }\end{array}$ \\
\hline
\end{tabular}

